\title{
Analysis of 5 Rare Cases of Esophageal Fistula after Anterior Cervical Decompression, Bone Graft Fusion and Internal Fixation Surgeries
}

\author{
Yu Huang \\ The First Affiliated Hospital of Fujian Medical University \\ Jin Lin \\ Fujian Health College \\ Lihan Zhou \\ The First Affiliated Hospital of Fujian Medical University \\ Jianhua Lin \\ The First Affiliated Hospital of Fujian Medical University \\ Xuanwei Chen ( $\sim$ 362024031@qq.com) \\ The First Affiliated Hospital of Fujian Medical University
}

\section{Research article}

Keywords: Esophageal fistula, Cervical spine, Anterior cervical approach

Posted Date: March 17th, 2020

DOI: https://doi.org/10.21203/rs.3.rs-17451/v1

License: (c) (1) This work is licensed under a Creative Commons Attribution 4.0 International License. Read Full License 


\section{Abstract}

Objective: To retrospectively analyze and discuss the characteristics of the pathogenesis, diagnosis and treatment of esophageal fistula after anterior cervical surgery.

Methods: A retrospective analysis of the clinical data of 5 patients complicated with esophageal fistula after anterior cervical surgery in our hospital from January 2003 to December 2018 was performed.

Results: Of the 5 patients, 3 died of severe pulmonary infection and sepsis after conservative treatment, and 2 patients healed after surgery performed for debridement and internal fixator removal.

Conclusion: Esophageal fistula after anterior cervical spine surgery is a rare and serious complication with a high mortality rate. Surgical debridement and removal of the internal fixator accompanied by other active treatment measures can achieve good curative effects and significantly improve the outcomes of patients.

\section{Background}

Esophageal fistula is a rare but serious complication after anterior cervical surgery and was first reported by Balmaseda in $1985^{[1]}$. The incidence of esophageal fistula after anterior cervical surgery is estimated to be $0.04 \% \sim 0.25 \%$ according to the previous relevant literature ${ }^{[2-6]}$. Despite a relatively low incidence, esophageal fistula may result in serious consequences, such as wound infection in mild cases, mediastinal infection, pulmonary infection, and sepsis in severe cases, and even death in the most serious cases. We analyzed the clinical data of 5 patients with esophageal fistula who underwent anterior cervical surgery in our hospital from 2003 to 2018 and discussed the causes, diagnosis and treatment of esophageal fistula.

\section{Materials And Methods}

1. General Data: From 2003 to 2018, there were 2,789 cases of anterior cervical spine surgery with follow-up data in our hospital. Among these cases, 5 patients developed esophageal fistula, and the incidence rate of esophageal fistula was $0.18 \%$. The 5 patients with esophageal fistula after operation were all male, aged 22-74 years old, with an average age of 47.6 years old. Among the 5 patients, patient 4 was diagnosed with cervical spinal canal stenosis, patient 3 was diagnosed with cervical spine fracture, patients 2 and 5 were diagnosed with cervical vertebra fracture with paralysis, and patient 1 was diagnosed with cervical vertebra dislocation with incomplete paralysis (as shown in Table 1).

2. Clinical Manifestations: According to the onset time, the disease can be divided into three classifications: intraoperative, early postoperative (within 30 days after surgery) and delayed esophageal fistula (> 30 days after surgery) ${ }^{[7]}$. There were no intraoperative esophageal fistulas or delayed esophageal fistulas in the 5 patients. Early esophageal fistulas were found in all 5 patients 3-8 days after their operations. Among them, patient 4 presented an increased drainage volume in the operation cavity that increased obviously when drinking water one day after the operation, patient 3 showed dysphagia 3 days after the operation, and patients 1,2 and 5 showed incision swelling and pain within 3 days after the removal of the drainage tube, and purulent secretions appeared at the drainage orifice and incision site.

3. Diagnosis: In addition to the clinical manifestations, the main diagnostic approaches of this group of patients include the following: (1) Oral administration of methylene blue solution: After oral administration, methylene blue solution can be exuded from the incision or the drainage tube opening. All 5 patients were given oral methylene blue solution, and methylene blue was seen exuding from the incision or from the drainage tube opening. (2) Gastroscopy: Among the 5 patients, considering their conditions, patients 2, 3 and 4 were treated with gastroscopy. Gastroscopy revealed esophageal fistula in all 3 patients; patients 2 and 3 presented only esophageal mucosal damage, and the underlying metal internal fixator was directly observed through the esophageal fistula in patient 4 (as shown in Figure 1a, 1b, 1c). (3) Esophagogram: Patient 1 underwent an esophagogram, but no obvious contrast agent leakage was observed (as shown in Figure 2a, 2b). However, the presence of gas shadow near the surgical segment of the cervical spine in positive and lateral cervical radiographs (as shown in Figure 2c, 2d), and the presence of abnormal soft tissue density shadow by CT or MRI (as shown in Figure 3 ) should be highly suspected to be associated with esophageal fistula.

4. Therapeutic approaches: Once the symptoms of suspected esophageal fistula after operation were identified, fasting and drinking were forbidden immediately. A gastric tube and jejunal tube were placed, nasogastric enteral was implemented, and systemic anti-infection agent administration, fluid infusion, incision dressing changes and other treatments were carried out. Among the 5 patients, patients 2,4 and 5 received the conservative treatment mentioned above, and patients 1 and 3 underwent another operation to remove the internal fixator 6-8 weeks after the initial cervical spine surgery.

5. Standard of healing: The standards of healing were as follows: (1) oral methylene blue did not flow out through the incision or drainage tube opening; (2) the fistula appeared healed under gastroscopy; (3) there was no obvious leakage of contrast agent in the esophagogram; and (4) the incision healed well.

\section{Results}

1. The clinical data of the 5 patients (as shown in Table 1).

Table 1. The clinical data of the 5 patients 


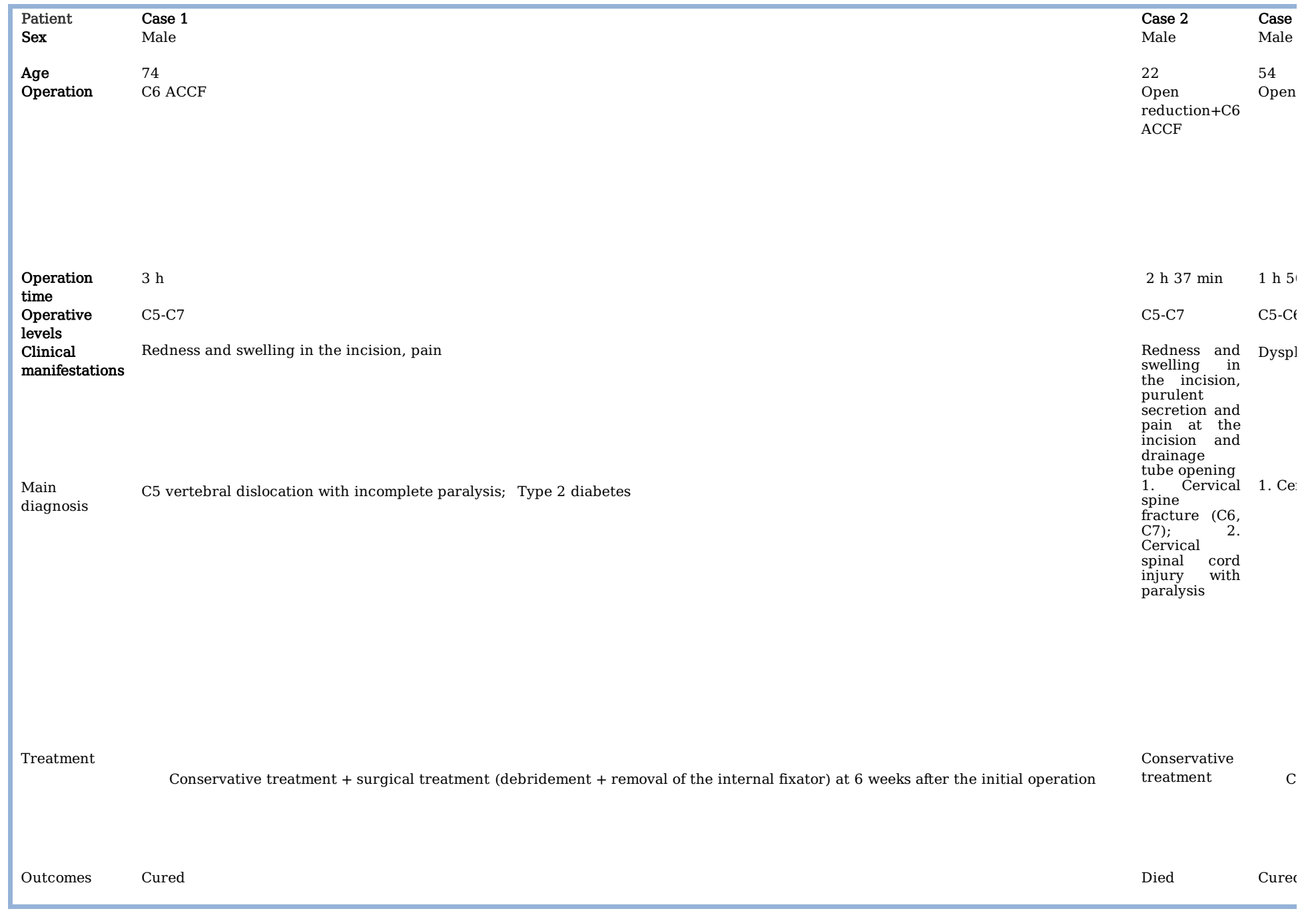

2. Outcome: Of the 5 patients in this study, patient 1 and 3 were cured after treatment and discharged from hospital; the 2 cured patients both healed after the second operation. The final outcome of patient2, 4 and 5 were death. The cause of death was considered to be severe pulmonary infection, septic shock, sepsis and systemic multiple organ failure after surgery.

\section{Discussion}

1. Incidence of esophageal fistula after anterior cervical surgery: Of the 2789 cases of anterior cervical surgery, a total of 5 patients developed esophageal fistula after anterior cervical surgery, with an incidence of $0.18 \%$. After Balmaseda first reported this complication in 1985, scholars locally and abroad have conducted relevant research on this disease. In 1989, Newhouse reported a total of 22 cases of esophageal fistula, with an incidence rate of $1.49 \%$ [5]. In 2000, Gaudinez $^{[8]}$ reported 44 cases, with an incidence rate of $0.25 \%$. In 2003 , domestic scholar Chen Xiongsheng ${ }^{[6]}$ reported 2 cases of postoperative esophageal fistula, with an incidence rate of $0.06 \%$, and in 2005 , the incidence rate reported by Jin Dadi ${ }^{[9]}$ was $0.24 \%$. In general, with the gradual clinical progression of anterior cervical surgery, the incidence of esophageal fistula after anterior cervical surgery is gradually decreasing, which is attributable to the improvements in surgical proficiency and the improvements in internal fixation materials.

2. Causes and high-risk factors: According to the relevant literature, the causes of esophageal fistula after anterior cervical surgery can be divided into three categories. The first category is iatrogenic injury. Early postoperative esophageal fistula is usually caused by the compression of the esophagus by surgical instruments for too long or the inadvertent injury of the esophageal tissue by sharp surgical instruments during the operation. The occurrence of late esophageal fistula is usually related to the loosening, dislodging and moving of internal fixators ${ }^{[3]}$. The second category is trauma. The incidence of esophageal fistula after cervical spine fracture is significantly higher than that associated with cervical degenerative diseases. Among 44 patients with esophageal fistula reported by Gaudinez ${ }^{[8]}, 34$ cases were caused by cervical spine fracture; esophageal fistula might be induced by inappropriate neck movement, falls and postoperative bronchoscopy or gastroscopy.The third category is surgical segment. Esophageal fistula complications after anterior cervical surgery occur mostly in patients who undergo operations of the C3-C6 segments and is related to the anatomical factors of the esophagus.

Anatomically, the esophageal wall is thin (only 3-4 mm in thickness); hence, mechanical entrapment easily induces ischemia and necrosis in this region ${ }^{[10]}$. Approximately $50 \%$ of esophageal injuries are reported to occur in the cervical segments associated with the esophagus, which is mainly composed of striated muscle with a mucosal lining and no serosa on the surface ${ }^{[11]}$. In addition, the upper and lower triangles of the pharyngeal muscles in the posterior wall of the 
esophagus (Killian's triangle and Laimer's triangle) are the most vulnerable regions of the esophagus to disease and the most common site of cervical esophageal injury (as shown in Figure4). Additional high-risk factors are as follows: elderly patients, patients with a poor general condition of the whole body; patients with complications of other basic diseases that are not conducive to postoperative recovery; and patients with a history of previous neck surgery are all at high risk for the onset of esophageal fistula.Among the 5 patients included in this study, there was no intraoperative esophageal fistula and no obvious evidence of operative injury or improper operation. After consideration, the possible high-risk factors for esophageal fistula development in this study may be

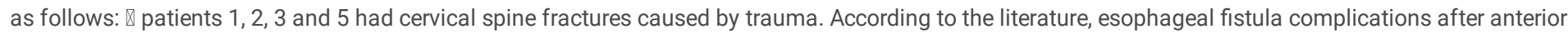
cervical spine surgery mostly occur in patients with cervical spine fractures ${ }^{[12-15]}$. Consequently, cervical spine fracture was an important factor in the occurrence of esophageal fistula in these 4 patients. Chronic or blunt injury to the esophagus in the process of cervical spine fracture caused by trauma can initiate the development of esophageal fistula in the thin posterior wall of the esophagus. $\otimes$ The cervical spine segments that were injured and operated on were located on the relatively weaker site of the esophageal wall in all 5 patients. $\otimes$ Patients 3 and 5 had cervical spine fractures combined with ankylosing spondylitis, which had a long disease course, and the patients had not undergone systematic treatment. According to the pathological characteristics of ankylosing spondylitis, the bone usually becomes brittle and hard, and the compact and sharp fractured section and small fracture fragments easily damage the esophagus when a fracture occurs. When fracture occurs, the compact and sharp fractured section and small fracture fragments may result in a high risk of damage to the esophagus ${ }^{[16]}$. Furthermore, patient 1 was diagnosed with type 2 diabetes after admission, with no systematic diagnosis and treatment in the past, and presented poorly controlled blood glucose levels. During hospitalization, the blood glucose level of the patient fluctuated between 14.5 and 9.1 $\mathrm{mmol} / \mathrm{L}$, which was considered high. The lack of effective blood glucose control in patients with diabetes is a significant factor in the poor healing of surgical incisions and fistulas ${ }^{[17]}$. Patient 4 had a history of cervical spine trauma and internal fixator implantation 2 years previously. The reason for this admission was that he fell and suffered another neck injury. Intraoperative exploration of the cervical internal fixation and esophageal space revealed purulent secretions, and an intraoperative diagnosis of "vertebral osteomyelitis" (delayed infection after cervical spine surgery) was made. The existence of a previous long-term metal internal fixator and inflammation, as well as scar formation in the operated area, can all cause tissue damage in the posterior esophageal wall, which is a high-risk factor associated with esophageal fistula ${ }^{[18]}$.

3. Diagnosis: (1) Clinical manifestations: The earliest manifestations are usually dysphagia, redness and pain at the incision, increased drainage volume, and purulent secretion at the incision and drainage orifice. However, the above clinical manifestations are not unique in postoperative patients with esophageal fistula. Nevertheless, it should be noted that a sudden increase in drainage fluid after drinking water or the leakage of food residue after eating can be regarded as specific manifestations of esophageal fistula after anterior cervical surgery. When inflammation cannot be controlled, inflammation quickly develops along the mediastinum, which may further lead to mediastinal infection, pulmonary infection, and even sepsis and multiple organ failure, eventually leading to death. (2) Auxiliary examinations and tests: $\nabla$ Oral administration of methylene blue solution: Oral methylene blue administration is the simplest and most accurate test in the early stage of suspected esophageal fistula and before relevant imaging examinations are performed. $\otimes$ Positive and lateral $X$ rays of the cervical spine: The main manifestations of esophageal fistula on positive and lateral X-rays of the cervical spine are subcutaneous edema of the neck, changes in the anterior space of the vertebral body, and displacement of the internal fixator and bone graft. However, the sensitivity of $\mathrm{X}$-ray examination for the diagnosis of esophageal fistula was only $72 \%\left[{ }^{[9]}\right.$. $\otimes$ Digital upper gastrointestinal radiography: Digital upper gastrointestinal radiography can directly confirm the occurrence of esophageal fistula, and the diffusion range of the contrast agent can help to determine the location of the esophageal fistula and the extent of purulent secretion diffusion. $\otimes$ CT and MRI: CT and MRI can be used to evaluate the size of the abscess cavity and show the status of the internal fixator, bone graft and surrounding bone structure. According to the research of White ${ }^{[19]}$, the gas outside the esophageal leak is the most definite atypical clinical manifestation of esophageal leakage. Additionally, MRI is the gold standard for diagnosis when esophageal fistula is accompanied by vertebral body infection; its sensitivity is $96 \%$, and its specificity can be as high as $93 \%{ }^{[20]}$. However, it is necessary to carefully determine whether the patient can tolerate a prolonged CT or MRI examination. $\otimes$ Gastroscopy: The position and surrounding conditions of the fistula can be directly observed. However, this examination is not recommended for patients after internal cervical spine fixation because it can miss new posterior pharyngeal wall and esophageal fistulas caused by reinjury of the posterior pharyngeal wall and esophagus due to improper operation ${ }^{[21]}$. Additionally, the sensitivity of gastroscopy is only $64 \%$, despite a high specificity ${ }^{[9]}$. According to relevant research reports, inflammation of the esophagus during endoscopy may lead to adverse consequences, such as the spread of infection in the mediastinum and enlargement of the esophageal fistula ${ }^{[22]}$. In general, the oral administration of methylene blue solution can be adopted first in the case of suspected postoperative esophageal fistulas. Esophageal fistula can be diagnosed initially if the methylene blue solution exudes from the incision. Then, further examinations, such as gastroscopy, can be selected according to the patient's condition.

4. Treatment: On the basis of relevant reports, there are still large differences in the treatment of postoperative esophageal fistula. On the one hand, according to the opinions of some experts and scholars who support conservative treatment, patients with esophageal fistula after cervical anterior cervical surgery can be treated by solid and liquid fasting, active dressing changes, intravenous antibiotic administration, indwelling of a gastric tube, and nasogastric enteral nutrition combined with intravenous nutrition to achieve natural fistula healing. On the other hand, some experts and scholars who support surgical treatment think much attention should be paid to the nonhealing of the fistula caused by the compression of tissues around the fistula by the internal fixator. Surgical removal of the internal fixator and thorough debridement of the operative cavity may be performed if the patient's basic conditions allow. Timely operation may contribute to the healing of the esophageal fistula ${ }^{[21]}$. In the patients in this study, two were treated conservatively for 6-8 weeks, but the fistula did not heal. They were treated with "debridement + removal of the internal cervical vertebra fixator". Intraoperative exploration showed screw loosening and plate displacement, obvious compression by the displaced plate on the posterior wall of esophagus, and marked inflammation in the tissues around the plate. Esophageal fistula repair was not performed due to the small size of the fistula. The operation proceeded successfully, and the patients were treated continuously with nasogastric enteral nutrition combined with intravenous nutrition postoperatively. After surgery, the incision exudation was alleviated gradually. Finally, the 2 patients were discharged from the hospital with esophageal fistula healing confirmed by the oral administration of methylene blue solution, upper digestive tract digital radiography and electronic gastroduodenoscopy (as shown in Figure 1d). However, due to pulmonary infections, the other 3 patients who underwent conservative treatment showed gradual aggravation of the infections and eventually developed severe pneumonia, sepsis, shock, multiple organ failure and finally death despite treatments with intravenous antibiotics. 
We analyzed the treatment process of the three patients as well as relevant literature reports. In the case of the 3 patients who actively received intravenous systemic antibiotic therapy, the infection of multiple organs was not alleviated and even became incrementally worse. We consider that the reason for the above situation was the long-term existence of infection around the cervical esophageal fistula, which caused the systemic intravenous antibiotic therapy to fail at achieving satisfactory results. However, according to the literature reports, patients complicated with esophageal fistula after anterior cervical surgery still have a $20-25 \%$ probability of abscess formation after conservative treatment, and the final mortality rate can reach $18 \%[23,24]$. Therefore, we advocate active treatment for patients complicated with esophageal fistula after anterior cervical surgery.

In patients with esophageal fistula complications after anterior cervical surgery, the fistula does not heal for a long time, and secondary surgery should be performed as soon as possible to remove the internal fixator. The main reasons for active surgical treatment are as follows: (1) The metal internal fixator will exert a certain pressure on the posterior wall of the esophagus that may cause ischemia of the fistula tissue and lead to poor blood supply in the fistula. Under low blood supply conditions in the tissue around the fistula, long-term nonhealing or even further expansion of the fistula may occur. (2) The internal fixator is generally a foreign metal body, and patients may present different degrees of rejection to the metal internal fixator. When such stimulation exists for a long time, it will often lead to a persistent fistula ${ }^{[25]}$. (3) The metal internal fixator very easily becomes the site of bacterial colonization in cases of incision and cavity infections. Timely removal of the internal fixator is helpful to promote infection control and is beneficial for systemic anti-infection treatments.

Therefore, after a certain period of conservative treatment, when fistula healing is poor, nonexistent or becomes worse, surgical conditions should be implemented for the patient as soon as possible. The compression and stimulation of the posterior esophageal wall by the internal fixator can be relieved by removing the metal internal fixator, which is often a steel plate, and the surgical cavity should be thoroughly washed and debrided during the operation. The existing infection should be removed as much as possible, and the neck should be externally fixed with a neck brace after the second operation. Active surgical treatment can obviously promote the healing of esophageal fistula.

In conclusion, esophageal fistula after anterior cervical surgery is a rare but serious postoperative complication with extremely high mortality. The mortality rate in this group of patients was $60 \%$, while according to reports from domestic and foreign studies, the mortality rate of esophageal fistula after anterior cervical surgery is $9-45 \%{ }^{[13]}$. Because its clinical manifestations sometimes lack specificity, it is necessary to find, diagnose and treat it as early as possible. Regarding treatment, through the analysis of the diagnosis and treatment of the 5 rare cases in this group, we suggest adopting a more active surgical treatment scheme as much as possible and promoting the healing of esophageal fistula through the removal of internal fixators and thorough debridement. Active surgical treatment is important for curing esophageal fistula and improving the prognosis of anterior cervical surgery.

\section{References}

1. MT Balmaseda, DJ Pellioni. Esophagocutaneous fistula in spinal cord injury: a complication of anterior cervical fusion[J]. Arch Phys Med Rehabil, 1985, 66(11): 783-784

2. Linqin Cai, Shaobo Wang, Mai Li, et al. Prevention and treatment of surglcal complications caused by operation of cervical spondylosis [J].Chinese Journal of Spine and Spinal Cord, 1995, 5(5):200-202.

3. Shaobo Wang, Shenglin Wang, Qingjun Ma囚et al. Diagnosis and treatment of esophageal fistula in anterior cervical spine surgery [J]. Chinese Journal of

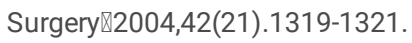

4. Guizhen Li, Jian Ma, et al, Clinical analysis of esophageal fistula complicated by anterior cervical surgery [J]. World Latest Medicne Information, 2016, $89: 406$

5. New house KE, Lindersey RW, Clark C R. Esophageal perforation following anterior cervical spine su rgery [J].S pine , 1989 , 14:1051-1052.

6. Xiongsheng Chen, Lianshun Jia, Shifeng Cao, et al. Prevention and management of complications in anterior cervical spine surgery[J].Chinese Journal of Orthopaedics, 2003®23(11):644-649.

7. Halani SH, Baum GR, Riley JP, et al. Esophageal perforation after anterior cervical spine surgery: a systematic review of the literature [J]. J Neurosurg Spine, 2016, 25(3): 285-291.

8. Gaudinez RF, English GM , Gebhard JS , et al.Esophageal perforations after anterior cervical surgery [J] .J Spinal Disord , 2000 ,13:77-84

9. Dadi Jin, Jian Wang, Dongbin Qu,et al, Analysis of early complications in anterior cervical spine surgery[J]. Chinese Journal of Orthopaedics, 2005, 25(2): 102ख106.

10. Ye Lin, Zhenyu Li, Sanqing Zhu, Analysis of complications and related problems in anterior cervical surgery [J]. The Orthopedic Journal of China,1998, 5:40-41.

11. Brinster C J, Singhal S, Lee L, et al. Evolving options in the management of esophageal perforation[ J] . Ann Thorac Surg, $2004,77: 1475-1483$.

12. Chen JY, Chen WJ, Huang TJ.S pinal epidural abscess complicating cervical spine fractu re with hypopharyngeal perforation [J].Spine, 1992 , 17:971-974.

13. Qingjun Ma, Gengting Dang, Qinlin Cai, et al,Esophageal fistula after anterior cervical surgery [J].Chinese Journal of Spine and Spinal Cord, 1998 , 8:110111.

14. Kriskovich MD, Apf elbaum RI , Haller JR.Vocal fold paralysis after ant erior cervi cal spine surgery :incidence, mechanism , and prevention of injury[J].Laryngoscope, 2000, 110:1467-1473

15. Nerot C , Jeanneret B , Lardenois T, et al. Esophageal perforation after fracture of the cervical spine: case report and review of the literature [J]. J Spinal Disord Tech, 2002, 15:513-518

16. Chunguang Zuo, Jun Zhang, Xinhu Wang, et al, A case report of esophageal fistula secondary to cervical fracture and dislocation in ankylosing spondylitis [J]. Chinese Journal of Spine and Spinal Cord, 2010, 20(9);715 
17. Hongli Ma, Risk factors and strategy for surgical incision infection in department of abdominal surgery[J]. Chinese Journal of Nosocomiology, 2011, 21(7):1310-1312.

18. Yong Liu, Lianshun Jia, et al. Diagnosis and treatment of esophageal fistula complicated by anterior cervical surgery [J]. Orthopedic Journal of China,2014,22(7):629-631

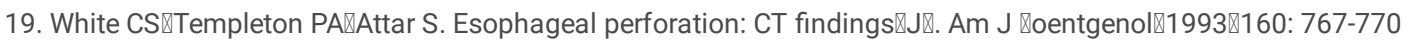

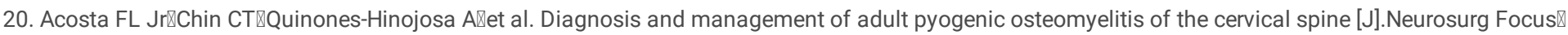
2004囚17(6): E2

21. Mardan Mamat, Alim Abdurexit, Sheng Weibin囚et al, Diagnosis and treatment of pharyngostoma and esophagostoma after anterior cervical spine surgery [J]. Chinese Journal of Orthopaedics,2016.9,36(17):1085-1092

22. Zhiyong Zhang, Jingguang Zhang , Yushang Cui , et al. Diagnosis and treatment of esopbageal perforation and disruption [J]. Chinese Journal of Gastrointestinal Surgery, 2003, 6(5).

23. Lu X, Guo Q, Ni B. Esophagus perforation complicating anterior cervical spine surgery [J]. European Spine Journal, 2012, 21(1):172-177.

24. Reid R R, Dutra, José, Conley D B, et al. Improved repair of cervical esophageal fistula complicating anterior spinal fusion: free omental flap compared with pectoralis major flap [J]. Journal of Neurosurgery: Spine, 2004, 100(1): 66-70.

25. Lin Sun, Yueming Song, et al. Management of esophageal fistula caused by anterior cervical spine surgery [J]. Chinese Journal of Orthopaedics, 2012, 32(10): 906-910

\section{Declarations}

\section{Acknowledgments}

Not applicable.

\section{Funding}

Not applicable.

\section{Availability of data and materials}

Data supporting the results reported in a published article can be found. Please contact author for data requests.

\section{Authors' contributions}

The authors' contributions to this study were as follow: study design, YH, JL, JHL, and LHZ; data collection, YH, JL; statistical analysis YH, JL, andXWC; manuscript writing by all authors. None of authors had a personal or financial conflict of interest.

\section{Competing interests}

The authors declare that they have no competing interests.

\section{Consent for publication}

Not applicable

\section{Ethics approval and consent to participate}

This study was approved by the ethics review board in the First Affiliated Hospital of Fujian Medical University, Fuzhou, China. No patient consent was obtained as this is a retrospective study based on clinical records.

\section{Abbreviations}

MRI: Magnetic resonance imaging

CT囚Computed Tomography

\section{Figures}



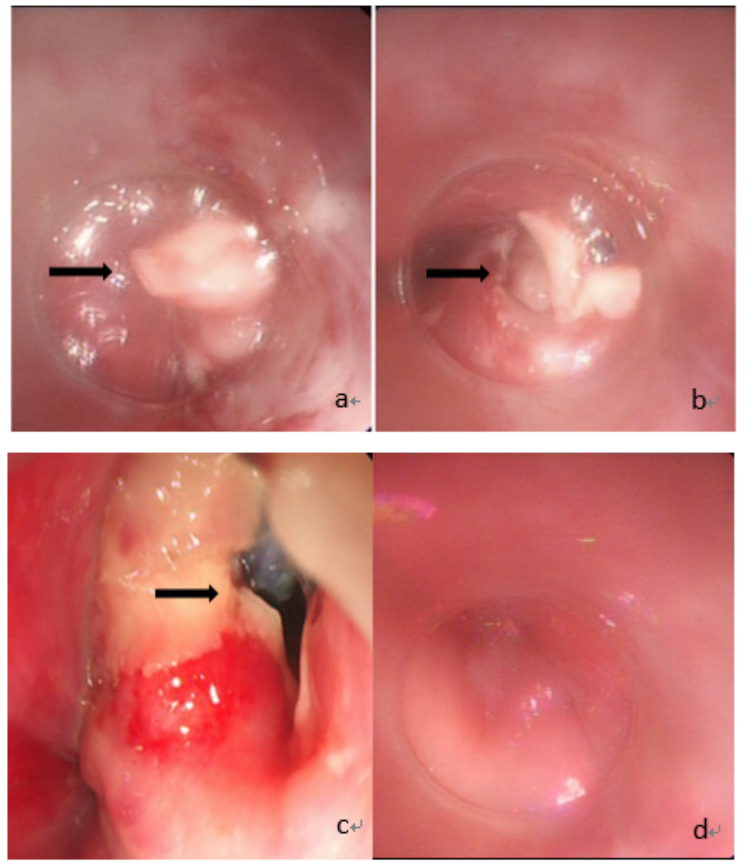

Figure1. The gastroscopy comparison of case 3 before (a, b) and after (d) the operation showed the healing of esophageal fistula; The gastroscopy of case4 show metal internal fixator (d).

Figure 1

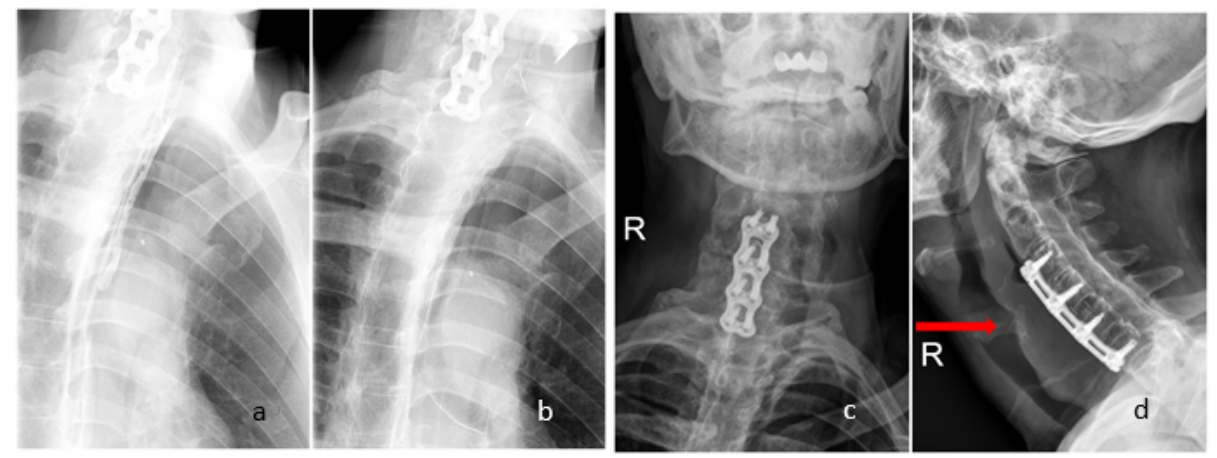

Figure 2. The esophagogram of case $3(\mathrm{a}, \mathrm{b})$ showed no obvious leakage of the contrast agent; Positive(c) and lateral(d) X-ray of case 3 showed enlargement of the anterior space of the vertebral body(red arrow).

Figure 2 


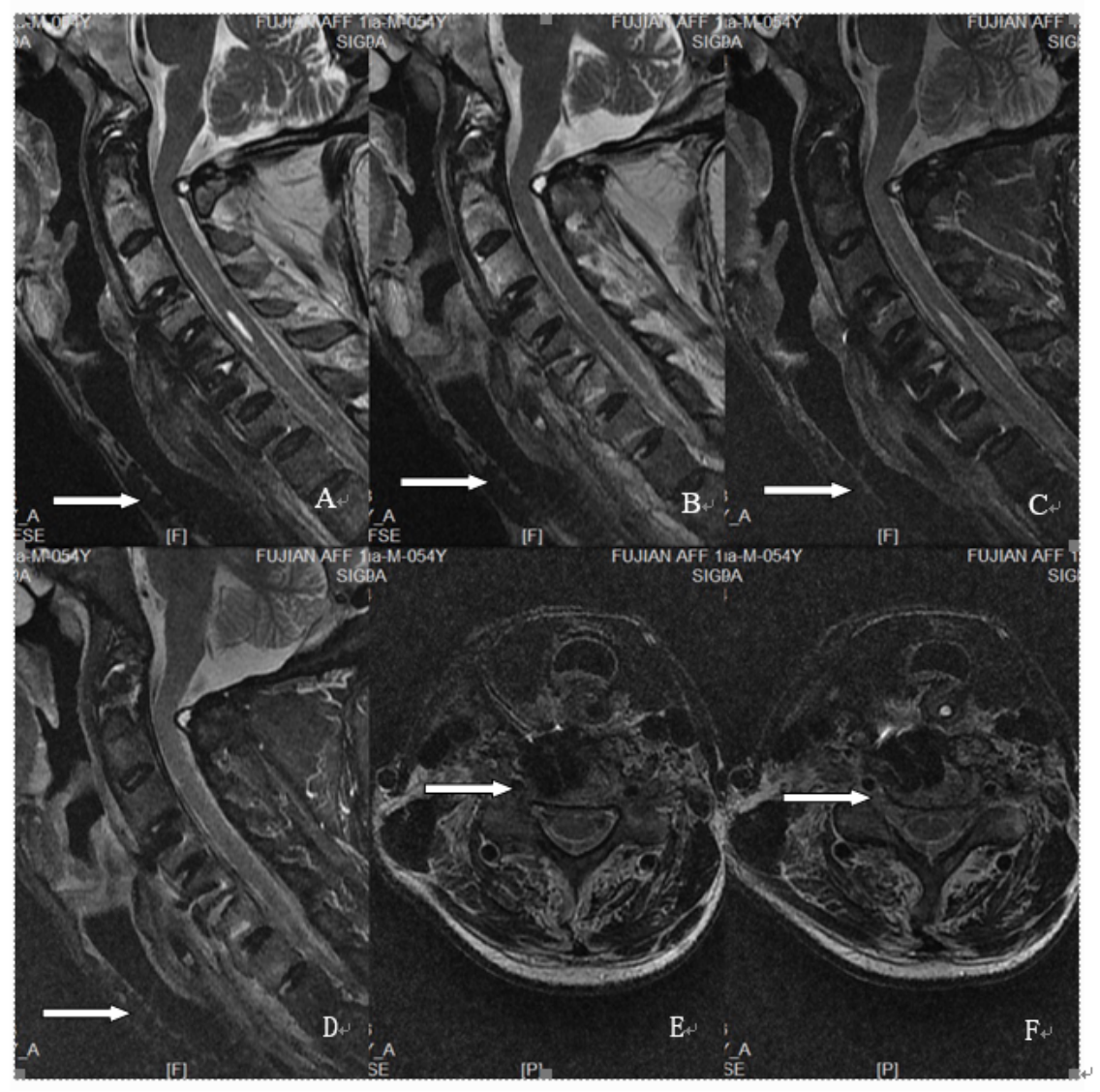

Figure 3. The MRI of case 3 (A-F) showed an abnormal-density shadow (white arrow) composed of soft tissue around the anterior part of the operated cervical spine segment.

\section{Figure 3}

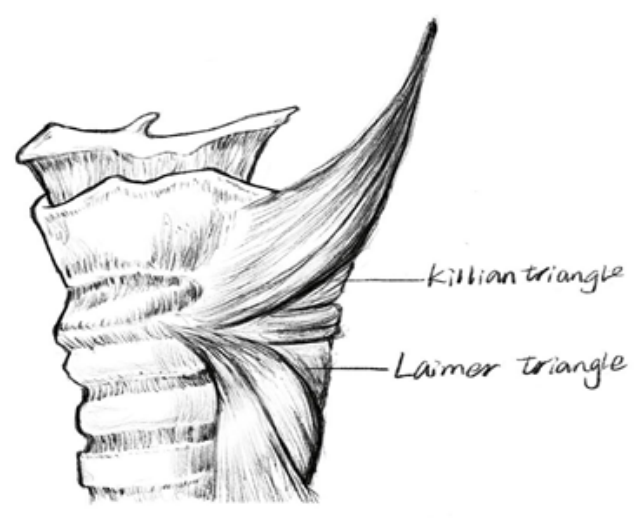

Figure 4. The anatomy of Killian's triangle and Laimer's triangle (painted by hand by the author).

\section{Figure 4}

